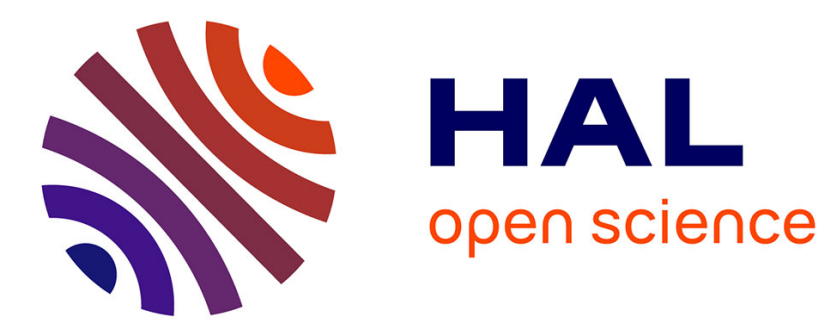

\title{
Efficient services composition for grid-enabled data-intensive applications
}

\author{
Tristan Glatard, Johan Montagnat, Xavier Pennec
}

\section{To cite this version:}

Tristan Glatard, Johan Montagnat, Xavier Pennec. Efficient services composition for grid-enabled data-intensive applications. High Performance and Distributed Computing, Jun 2006, Paris, France. pp.333-334, 10.1109/HPDC.2006.1652170 . hal-00683201

\section{HAL Id: hal-00683201 \\ https://hal.science/hal-00683201}

Submitted on 28 Mar 2012

HAL is a multi-disciplinary open access archive for the deposit and dissemination of scientific research documents, whether they are published or not. The documents may come from teaching and research institutions in France or abroad, or from public or private research centers.
L'archive ouverte pluridisciplinaire HAL, est destinée au dépôt et à la diffusion de documents scientifiques de niveau recherche, publiés ou non, émanant des établissements d'enseignement et de recherche français ou étrangers, des laboratoires publics ou privés. 


\section{Efficient services composition for grid-enabled data-intensive applications}

\author{
Tristan Glatard, Johan Montagnat \\ I3S, CNRS \\ \{glatard,johan\}@i3s.unice.fr
}

\author{
Xavier Pennec \\ INRIA, Epidaure \\ Xavier.Pennec@sophia.inria.fr
}

\begin{abstract}
The problem we address in this paper is to build complex applications by reusing and assembling scientific codes on a production grid infrastructure. We first review the two main paradigms for executing application code on a grid: (a) the task based approach, associated to global computing, characterized by its efficiency, and (b) the service approach developed in the meta computing and the Internet communities, characterized by its flexibility. We carefully introduce the terminologies coming from different computing coтmunities and we combine the advantages of both approaches.

We argue that the service approach is more relevant for composing application codes. However, (i) it introduces an extra level of complexity on the application developer side and (ii) it makes application optimization on a grid more difficult. We propose solutions based on (i) a generic code wrapper which simplifies new application services development and (ii) a new grouping strategy coupled with traditional data and services parallelization techniques, that proved to be underused in the grid area for optimization. These solutions are implemented in our grid workflow enactor prototype. To our knowledge, it is the first implementation taking into account all these optimization levels.

The theoretical performances are analyzed and compared to real measurements. Results are shown on a real, data-intensive application to medical images analysis. The execution is done on the very large scale EGEE production infrastructure. An execution time speed up of approximately 9 is achieved, with a minimal additional cost for application developers. The strategies adopted being generic, many other applications could similarly be instrumented at very low cost. Moreover, our optimized application enactor is based on existing standards and it is freely available for download.
\end{abstract}

\section{Introduction}

As a consequence of the tremendous research effort carried out by the international community these last years and the emergence of standards, grid middlewares have reached a maturity level such that large grid infrastructures where deployed (EGEE [8], OSG [24], NAREGI [21]) and sustained computing production was demonstrated for the benefit of many industrial and scientific applications [19]. Yet, current middlewares expose rather low level interfaces to the application developers and enacting an application on a grid often requires a significant work involving computer and grid system experts.

Considering the considerable amount of sequential, non grid-specific algorithms that have been produced for various data processing tasks, grid computing is very promising for:

- Performing complex computations involving many computation tasks (codes parallelism).

- Processing large amounts of data (data parallelism).

Indeed, beyond specific parallel codes conceived for exploiting an internal parallelism, grids are adapted to the massive execution of different tasks or the re-execution of a sequential code on different data sets which are needed for many applications. In both cases, temporal and data dependencies may limit the parallelism that can be achieved.

To handle user processing requests, two main strategies have been proposed and implemented in grid middlewares:

1. In the task based strategy, also referred to as global computing, users define computing tasks to be executed. Any executable code may be requested by specifying the executable code file, input data files, and command line parameters to invoke the execution. The task based strategy, implemented in GLOBUS [9], LCG2 [16] or gLite [13] middlewares for instance, has already been used for decades in batch computing. It makes the use of non grid-specific code very simple, provided that the user has a knowledge of the exact syntax to invoke each computing task.

2. The service based strategy, also referred to as meta computing, consists in wrapping application codes into standard interfaces. Such services are seen as black boxes from the middleware for which only the invocation interface is known. Various interfaces such as Web 
Services [30] or gridRPC [20] have been standardized. The services paradigm has been widely adopted by middleware developers for the high level of flexibility that it offers (OGSA [10]). However, this approach is less common for application code as it requires all codes to be instrumented with the common service interface.

In this paper, we are interested in building data-intensive applications by composing data processing algorithms. We are proposing efficient and transparent grid processing of such applications on grid infrastructures through an optimized workflow manager. We discuss the strengths of the service based approach for achieving this goal in section 2 and we present execution optimizations in section 3 . The workflow manager is evaluated on a realistic application to medical images registration in section 4 . The services composition tool proposed is generic and reusable. Many application could thus be instrumented at a low cost from the application developer's point of view.

\section{Composing complex applications}

Many sequential codes have been and are being developed for data analysis. Building applications by assembling such algorithms is very common, in the image processing field for instance, where an image analysis procedure often involves a sequence of basic image processing operations. In many other application areas, such application composition is praised as it allows code reusability without introducing a too high load on the application developers.

The task based approach has been used for grid and batch computing for a very long time. The service based approach is more dynamic and flexible but it is usually used for accessing remote resources which do not necessarily benefit from grid computing capabilities. This is acceptable for most middleware services that are located and executed on a single server but application services that may require compute-intensive code execution and that are invoked concurrently in the context of the target applications, can easily overwhelm the computing capabilities of a single host. To overcome these limitations some approaches have been explored, such as submission services replacing the straight task submission [11] or generic services for wrapping any legacy code with a standard interface [15].

\subsection{Composing services for building appli- cations}

When building an application by composing basic processings, the application logic, referred to as the application workflow, can intuitively be represented through a directed graph of processors (graph nodes) representing computation jobs and data dependencies (graph arrows) constraining the order of invocation of processors (see figure 1).

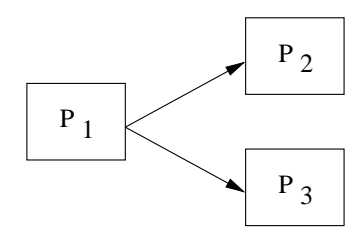

\section{Figure 1. Simple workflow example.}

Many workflow representation formats and execution managers have been proposed in the literature with very different capabilities [31]. We are especially targeting dataintensive workflow managers used for scientific applications.

In the task based approach, the description of a task, or computation job, encompasses both the processing (binary code and command line parameters) and the data (static declaration). Workflow processors directly represent computing tasks. The user is responsible for providing the binary code to be executed and for writing down the precise invocation command line. All computations to be performed are statically described in the graph.

Conversely in the service based approach, the input data are treated as input parameters (dynamic declaration), and the service appears to the end user as a black box hiding the code invocation. This difference in the handling of data (static or dynamic declaration) makes the application composition far easier from a user point of view, as detailed in section 2.2. The service based approach is also naturally very well suited for chaining the execution of different algorithms assembled to build an application. Indeed, the interface to each application component is clearly defined and the middleware can invoke each of them through a single protocol.

In a service based workflow, each processor is representing an application component. In addition to the processors and the data arrows, a service based workflow representation requires a number of input and output ports attached to each processor. The oriented arrows are connecting output ports to input ports. Two special processor nodes are defined: data sources are processors without input ports (they are producing data to feed the workflow) and data sinks are processors without output ports (they are collecting data produced).

A significant difference between the service and task approaches of workflow composition is that there may exist loops in a service based workflow given that an input port can collect data from different sources as illustrated in figure 2. This kind of workflow pattern is common for optimization algorithms: it corresponds to an optimization loop 
converging after a number of iterations determined at the execution time from a computed criterion. In this case, the output of processor $P_{1}$ would correspond to the initial value of this criterion. $P_{3}$ produces its result on one of its two output ports, whether the computation has to be iterated one more time or not. On the contrary, there cannot be a loop in the graph of a task based workflow as this would mean that a processor input is depending on one of its output. An emblematic task-based workflow manager is indeed called Directed Acyclic Graph Manager (DAGMan) ${ }^{1}$. Composing such optimization loop would not be possible, as the number of iterations is determined during the execution and thus cannot be statically described.

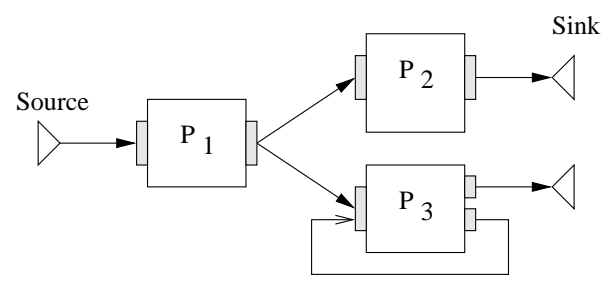

\section{Figure 2. Service-based workflow example.}

The service based approach has been implemented in different workflow managers. The Kepler system [18] targets many application areas from gene promoter identification to mineral classification. It can orchestrate standard Web-Services linked with both data and control dependencies and implements various execution strategies. The Taverna project [23], from the myGrid e-Science UK project $^{2}$ targets bioinformatics applications and is able to enact Web-Services and other components such as Soaplab services [25] and Biomoby ones. It implements high level tools for the workflow description such as the Feta semantic discovery engine [17]. Other workflow systems such as Triana [28], from the GridLab project ${ }^{3}$, are decentralized and distribute several control units over different computing resources. This system implements both a parallel and a peer-to-peer distribution policies. It has been applied to various scientific fields, such as gravitational waves searching [7] and galaxy visualization [27].

\subsection{Data-intensive grid-enabled applica- tions}

The main difference between the task based and the service based approach appears when considering the reexecution of the same application workflow over an input data set. In a task based workflow, a computation task is defined

\footnotetext{
${ }^{1}$ Condor DAGMan, http : / / www.cs.wisc.edu/condor/dagman/

${ }^{2}$ http://mygrid.org.uk

${ }^{3}$ http: //www.gridlab.org
}

\begin{tabular}{c|cc|c}
$\mathrm{A}$ & $\mathrm{B}$ & $\mathrm{A}$ & $\mathrm{B}$ \\
\hline $\mathrm{A}_{0}$ & $\mathrm{~B}_{0}$ & $\mathrm{~A}_{0}$ & $\mathrm{~B}_{0}$ \\
$\mathrm{~B}_{1}$ & & $\mathrm{~A}_{1}$ & $\mathrm{~B}_{1}$ \\
$\vdots$ & $\vdots$ & $\vdots$ & $\vdots$ \\
$\mathrm{A}_{\mathrm{n}}$ & $\mathrm{B}_{\mathrm{n}}$ & $\mathrm{A}_{\mathrm{n}}$ & $\mathrm{B}_{\mathrm{n}}$
\end{tabular}

Figure 3. Action of the cross product (left) and dot product (right) operators on the input data

by a single input data set and a single processing. Executing the same processing over two data results in two independent tasks. This approach enforces the replication of the execution graph for every input data to be processed.

To get closer to the service-based approach, a simple extension to the task based approach is to propose multiinput data tasks descriptions where a generic task can be described for a set of input data, resulting in the execution of multiple jobs: one per input data. However, it is far from being commonly available in today's production middlewares and it is often treated at the application level. Moreover, multi-input tasks cannot be used in a workflow where each task needs to be replicated for every input data.

The service based approach easily accommodates with input data sets. Data sources are sequentially delivering input data but no additional complexity of the application graph is needed. An example of the flexibility offered by the service-based approach is the ability to define different iteration strategies over the input data of a service. When a service owns two input ports or more, an iteration strategy defines the composition rule for the data coming from all input ports pairwise. Consider two input sets $\mathrm{A}=\left\{\mathrm{A}_{0}, \mathrm{~A}_{1}, \ldots, \mathrm{A}_{n}\right\}$ and $\mathrm{B}=\left\{\mathrm{B}_{0}, \mathrm{~B}_{1}, \ldots, \mathrm{B}_{m}\right\}$ to a service. The most common iteration strategy consists in processing each data of the first set with each data of the second set in their order of definition, thus producing $\min (n, m)$ result. This corresponds to the case where a sequence of pairs need to be processed. This iteration strategy is referred to as a dot product in the Taverna community [23]. Another common iteration strategy, called a cross product, consists in processing all input data from the first set with all input data from the second set, thus producing $m \times n$ results. Figure 3 illustrates these two strategies. Many other strategies could be implemented but we limit ourselves to these two ones that are sufficient for implementing most applications.

Using iteration strategies to design complex data interaction patterns is a very powerful tool for data-intensive application developers. It makes the task replication problem associated to the task-based workflows combinatorial: a cross product produces an enormous amount of tasks and chaining cross products just makes the application work- 
flow representation intractable even for a limited number (tens) of input data. Despite the availability of graphical tools to design application workflow, dealing with many input data quickly becomes impossible for users.

\subsection{Data synchronization barriers}

A particular kind of processors are algorithms that need to take into account the whole input data set in their processing rather than processing each input one by one. This is the case for many statistical operations computed on the data, such as the computation of a mean or a standard deviation over the produced results for instance. Such processors are referred to as synchronization processors has they represent real synchronization barriers, waiting for all input data to be processed before being executed.

\subsection{Interfaces and platforms}

Another strength of the service based approach is to easily deal with multiple execution platforms. Each service is called as a black box without knowledge of the underlying platform. Several services may execute on different platforms transparently which is convenient when dealing with legacy code. In the task based approach, a specific submission interface is needed for each infrastructure.

\section{Scheduling and executing the services work- flow}

The service based approach is making services composition easier than the task based approach as discussed above. It is thus highly convenient from the end user point of view. However, in this approach, the control of jobs submissions is delegated to external services, making the optimization of the workflow execution much more difficult. The services are black boxes isolating the workflow manager from the execution infrastructure. In this context, most known optimization solutions do not hold.

Many solutions have indeed been proposed in the taskbased paradigm to optimize the scheduling of an application in distributed environments [6]. Concerning workflowbased applications, previous works [2] propose specific heuristics to optimize the resource allocation of a complete workflow. Even if it provides remarkable results, this kind of solutions is not directly applicable to the service-based approach. Indeed, in this latest approach, the workflow manager is not responsible for the task submission and thus cannot optimize the resource allocation.

Focusing on the service-based approach, nice developments such as the DIET middleware [5] and comparable approaches $[26,1]$ introduce specific strategies such as hierarchical scheduling. In [4] for instance, the authors describe a way to handle file persistence in distributed environments, which leads to strong performance improvements. However, those works focus on middleware design and do not include any workflow management yet. Moreover, those solutions require specific middleware components to be deployed on the target infrastructure. As far as we know, such a deployment has only been done on experimental platforms yet [3], and it is hardly possible for an application running on a production infrastructure.

Hence, there is a strong need for precisely identifying generic optimization solutions that apply to service-based workflows. In the following sections, we are exploring different strategies for optimizing the workflow execution in a service based approach, thus offering the flexibility of services and the efficiency of tasks. First of all, several level of parallelism can be exploited when considering the workflow execution for taking advantage of the grid computing capabilities. We describe them and then study their impact on the performances with respect to the characteristics of the considered application. Besides, we propose a solution for grouping sequential jobs in the workflow, thus allowing more elaborated optimization strategies in the service-based workflow area.

\subsection{Asynchronous services calls}

To enable parallelism during the workflow execution, multiple application services have to be called concurrently. The calls made from the workflow enactor to these services need to be non-blocking for exploiting the potential parallelism. GridRPC services may be called asynchronously as defined in the standard [20]. Web Services also theoretically enables asynchronous calls. However, the vast majority of existing web service implementations do not cover the whole standard and none of the major implementations [29, 14] do provide any asynchronous service calls for now. As a consequence, asynchronous calls to web services need to be implemented at the workflow enactor level, by spawning independent system threads for each processor being executed.

\subsection{Workflow parallelism}

Given that asynchronous calls are possible, the first level of parallelism that can be exploited is the intrinsic workflow parallelism depending on the graph topology. For instance if we consider the simple example presented in figure 2, processors $\mathrm{P}_{2}$ and $\mathrm{P}_{3}$ may be executed in parallel. This optimization is trivial and implemented in all the workflow managers cited above. 


\subsection{Data parallelism}

When considering data-intensive applications, several input data sets are to be processed using a given workflow. Benefiting from the large number of resources available in a grid, workflow services can be instantiated as several computing tasks running on different hardware resources and processing different input data in parallel.

Data parallelism denotes that a service is able to process several data sets simultaneously with a minimal performance loss. This capability involves the processing of independent data on different computing resources.

Enabling data parallelism implies, on the one hand, that the services are able to process many parallel connections and, on the other hand, that the workflow engine is able to submit several simultaneous queries to a service leading to the dynamic creation of several threads. Moreover, a data parallel workflow engine should implement a dedicated data management system. Indeed, in case of a data parallel execution, a data is able to overtake another one during the processing and this could lead to a causality problem, as we exemplified in [11]. To properly tackle this problem, data provenance has to be monitored during the data parallel execution. Detailed work on data provenance can be found in [32].

Consider the simple workflow made of 3 services and represented on figure 1. Suppose that we want to execute this workflow on 3 independent input data sets $D_{0}, D_{1}$ and $D_{2}$. The data parallel execution diagram of this workflow is represented on figure 4 . On this kind of diagram, the abscissa axis represents time. When a data set $D_{i}$ appears on a row corresponding to a processor $P_{j}$, it means that $D_{i}$ is being processed by $P_{j}$ at the current time. To facilitate legibility, we represented with the $D_{i}$ notation the piece of data resulting from the processing of the initial input data set $D_{i}$ all along the workflow. For example, in the diagram of figure 4 , it is implicit that on the $P_{2}$ service row, $D_{0}$ actually denotes the data resulting from the processing of the input data set $D_{0}$ by $P_{1}$. Moreover, on those diagrams we made the assumption that the processing time of every data set by every service is constant, thus leading to cells of equal widths. Data parallelism occurs when different data sets appear on a single square of the diagram whereas intrinsic workflow parallelism occurs when the same data set appears many times on different cells of the same column. Crosses represent idle cycles.

As demonstrated in the next sections, fully taking into account this level of parallelism is critical in service-based workflows, whereas it does not make any sense in taskbased ones. Indeed, in this case it is covered by the workflow parallelism because each task is explicitly described in the workflow description.

\begin{tabular}{|c||c|c|}
\hline & & $D_{0}$ \\
$P_{3}$ & $\mathrm{X}$ & $D_{1}$ \\
& & $D_{2}$ \\
\hline \multirow{1}{*}{$P_{2}$} & $\mathrm{X}$ & $D_{0}$ \\
& & $D_{1}$ \\
& & $D_{2}$ \\
\hline \multirow{3}{*}{$P_{1}$} & $D_{0}$ & \\
& $D_{1}$ & $\mathrm{X}$ \\
& $D_{2}$ & \\
\hline
\end{tabular}

Figure 4. Data parallel execution diagram of the workflow of figure 1

\begin{tabular}{|c||c|c|c|c|}
\hline$P_{3}$ & $\mathrm{X}$ & $D_{0}$ & $D_{1}$ & $D_{2}$ \\
\hline$P_{2}$ & $\mathrm{X}$ & $D_{0}$ & $D_{1}$ & $D_{2}$ \\
\hline$P_{1}$ & $D_{0}$ & $D_{1}$ & $D_{2}$ & $\mathrm{X}$ \\
\hline
\end{tabular}

\section{Figure 5. Service parallel execution diagram of the workflow of figure 1}

\subsection{Services parallelism}

Input data sets are likely to be independent from each other. This is for example the case when a single workflow is iterated in parallel on many input data sets.

Services parallelism denotes that the processing of two different data sets by two different services are totally independent. This pipelining model, very successfully exploited inside CPUs, can be adapted to sequential parts of servicebased workflows. Consider again the simple workflow represented in figure 1, to be executed on the 3 independent input data sets $D_{0}, D_{1}$ and $D_{2}$. Figure 5 presents a service parallel execution diagram of this workflow. Service parallelism occurs when different data sets appear on different cells of the same column. We here supposed that a given service can only process a single data set at a given time (data parallelism is disabled).

Data synchronization barriers, presented in section 2.3, are of course a limitation to services parallelism. In this case, this level of parallelism cannot be exploited because the input data sets are dependent from each other.

Here again, we show in the next section that service parallelism is of major importance to optimize the execution of service-based workflows. In task-based workflow, this level of parallelism does not make any sense because it is included in the workflow parallelism.

\subsection{Theoretical performance analysis}

The data and service parallelism described above are specific to the service-based workflow approach. To pre- 
cisely quantify how they influence the application performances we model the workflow execution time for different configurations. We first present general results and then study particular cases, making assumptions on the type of application run.

\subsubsection{Definitions and notations}

In the workflow, a path denotes a set of processors linking an input to an output. The critical path of the workflow denotes the longest path in terms of execution time.

$n_{W}$ denotes the number of services on the critical path of the workflow and $n_{D}$ denotes the number of data sets to be executed by the workflow.

$i$ denotes the index of the $i^{t h}$ service of the critical path of the workflow $\left(i \in\left[0, n_{W}-1\right]\right)$. Similarly $j$ denotes the index of the $j^{t h}$ data set to be executed by the workflow $\left(j \in\left[0, n_{D}-1\right]\right)$.

$T_{i, j}$ denotes the duration in seconds of the treatment of the data set $j$ by the service $i$. If the service submits jobs to a grid infrastructure, this duration includes the overhead introduced by the submission, scheduling and queuing times.

$\Sigma$ denotes the total execution time of the workflow

\subsubsection{Hypotheses}

The critical path is assumed not to depend on the data set. This hypothesis seems reasonable for most applications but may not hold in some cases as for example the one of workflows including algorithms containing optimization loops whose convergence time is likely to vary in a complex way w.r.t the nature of the input data set.

Data parallelism is assumed not to be limited by infrastructure constraints. We justify this hypothesis considering that our target infrastructure is a grid, whose computing power is sufficient for our application.

In this section, workflows are assumed not to contain any synchronization processors. Workflows containing such synchronization barriers may be analyzed as two sub workflows respectively corresponding to the parts of the initial workflow preceding and succeeding the synchronization barrier.

\subsubsection{Execution times modeling}

Under those hypotheses, we can determine the expression of the total execution time of the workflow for different execution policies:

- Sequential case (without service nor data parallelism):

$$
\Sigma=\sum_{i<n_{W}} \sum_{j<n_{D}} T_{i, j}
$$

- Case DP: Data parallelism only

$$
\Sigma_{D P}=\sum_{i<n_{W}} \max _{j<n_{D}}\left\{T_{i, j}\right\}
$$

- Case SP: Service parallelism only

$$
\Sigma_{S P}=T_{n_{W}-1, n_{D}-1}+m_{n_{W}-1, n_{D}-1}
$$

with: $\forall i \neq 0$ and $\forall j \neq 0$,

$$
m_{i, j}=\max \left(T_{i-1, j}+m_{i-1, j}, T_{i, j-1}+m_{i, j-1}\right)
$$

and:

$$
m_{0, j}=\sum_{k<j} T_{0, k} \quad \text { and } \quad m_{i, 0}=\sum_{k<i} T_{k, 0}
$$

- Case DSP: both Data and Service parallelism

$$
\Sigma_{D S P}=\max _{j<n_{D}}\left\{\sum_{i<n_{W}} T_{i, j}\right\}
$$

All the above expressions of the execution times can be demonstrated recursively. By lack of space, these proofs are not expanded here.

\subsubsection{Asymptotic speed-ups}

To better understand the properties of each kind of parallelism, it is interesting to study the asymptotic speed-ups resulting from service and data parallelism in particular application cases.

Massively data-parallel workflows. Let us consider a massively (embarrassingly) data-parallel application (single processor $\mathrm{P}_{0}$, very large number of input data). In this case, $n_{W}=1$ and the execution time is:

$\Sigma_{D P}=\Sigma_{D S P}=\max _{j<n_{D}}\left(T_{0, j}\right) \ll \Sigma=\Sigma_{S P}=\sum_{j<n_{D}} T_{0, j}$

In this case, data parallelism leads to a significant speedup. Service parallelism is useless but it does not lead to any overhead.

Non data intensive workflows. In such workflows, $n_{D}=$ 1 and the execution time is:

$$
\Sigma_{D S P}=\Sigma_{D P}=\Sigma_{S P}=\Sigma=\sum_{i<n_{W}} T_{i, 0}
$$

In this case, neither data nor service parallelism lead to any speed-up. Nevertheless, none of them does introduce any overhead. 
Data intensive complex workflows. In this case, we will suppose that $n_{W}>1$ and $n_{D}>1$. In order to analyze the speed-ups introduced by service and data parallelism, we make the simplifying assumption of constant execution times: $T_{i, j}=T$. The workflow execution time then resumes to:

$$
\begin{aligned}
\Sigma & =n_{D} \times n_{W} \times T \\
\Sigma_{D P}=\Sigma_{D S P} & =n_{W} \times T \\
\Sigma_{S P} & =\left(n_{D}+n_{W}-1\right) \times T
\end{aligned}
$$

If service parallelism is disabled, the speed-up introduced by data parallelism is:

$$
S_{D P}=\frac{\Sigma}{\Sigma_{D P}}=n_{D}
$$

If service parallelism is enabled, the speed-up introduced by data parallelism is:

$$
S_{D S P}=\frac{\Sigma_{S P}}{\Sigma_{D S P}}=\frac{n_{D}+n_{W}-1}{n_{W}}
$$

If data parallelism is disabled, the speed-up induced by service parallelism is:

$$
S_{S P}=\frac{\Sigma}{\Sigma_{S P}}=\frac{n_{D} \times n_{W}}{n_{D}+n_{W}-1}
$$

Service parallelism does not lead to any speed-up if it is coupled with data parallelism: $S_{S D P}=\frac{\Sigma_{D P}}{\Sigma_{D S P}}=1$. Thus, under those assumptions, service parallelism may not be of any use on fully distributed systems. However, section 4 will show that even in case of homogeneous input data sets, $\mathrm{T}$ is hardly constant in production systems because of the high variability of the overhead due to submission, scheduling and queuing times on such large scale and multi-user platforms. The constant execution time hypothesis does not hold. This appears to be a significant difference between grid computing and traditional cluster computing. Figure 6 illustrates on a simple example why service parallelism do provide a speed-up even if data parallelism is enabled, if the assumption of constant execution times does not hold. The left diagram does not take into account service parallelism whereas the right one does. The processing time of the data set $D_{0}$ is twice as long as the other ones on service $P_{0}$ and the execution time of the data set $D_{1}$ is three times as long as the other ones on service $P_{1}$. It can for example occur if $D_{0}$ was submitted twice because an error occurred and if $D_{1}$ remained blocked on a waiting queue. In this case, service parallelism improves performances beyond data parallelism as it enables some computations overlap. It justifies the experimental observations done in section 4 .
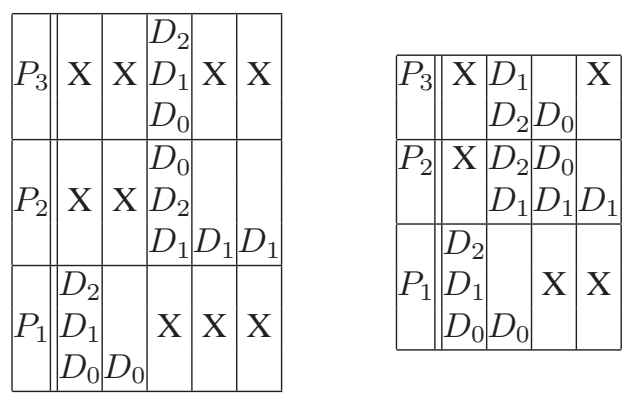

\section{Figure 6. Workflow execution time without (left) and with (right) service parallelism when the execution time is not constant.}

\subsection{Grouping services and targeting the ex- ecution platform}

In addition to the known strategies described so far, we propose a processors grouping strategy to further optimize the execution time of a workflow. Processors grouping consists in merging multiple jobs into a single one. It reduces the grid overhead induced by the submission, scheduling, queuing and data transfers times whereas it may also reduce the parallelism. In particular sequential processors grouping is interesting because those processors do not benefit from any parallelism. For example, considering the workflow of our application presented on figure 9 we can, for each data set, group the execution of the crestLines and the crestMatch jobs on the one hand and the PFMatchICP and the PFRegister ones on the other hand.

Grouping jobs in the task-based approach is straightforward and it has already been proposed for optimization [2]. Conversely, jobs grouping in the service-based approach is usually not possible given that (i) the services composing the workflow are totally independent from each other (each service is providing a different data transfer and job submission procedure) and (ii) the grid infrastructure handling the jobs does not have any information concerning the workflow and the job dependencies. Consider the simple workflow represented on the left side of figure 7. On top, the services for $P_{1}$ and $P_{2}$ are invoked independently. Data transfers are handled by each service and the connection between the output of $P_{1}$ and the input of $P_{2}$ is handled at the workflow engine level. On the bottom, $P_{1}$ and $P_{2}$ are grouped in a virtual single service. This service is capable of invoking the code embedded in both services sequentially, thus resolving the data transfer and independent code invocation issues. It breaks the hypothesis of all services seen as black boxes whose internal logic is unknown.

The solution we propose to enable job grouping in a service-based workflow is to use a generic submission ser- 


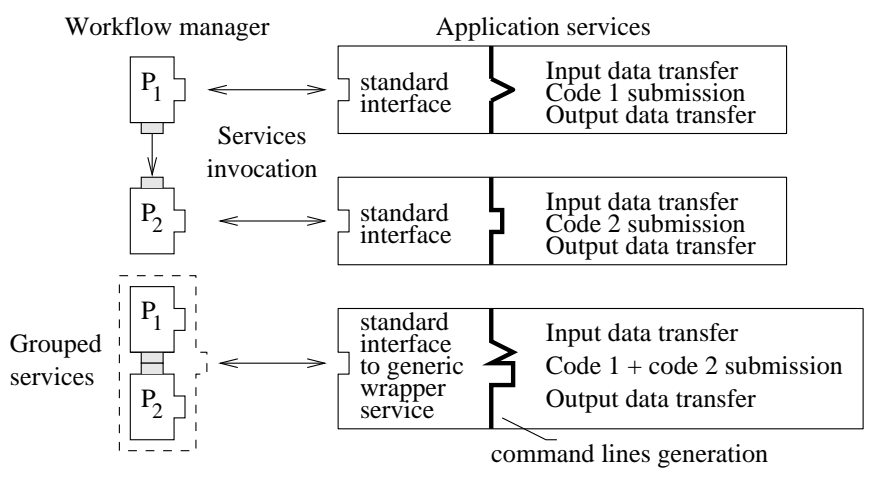

Figure 7. Classical services invocation (top) and services grouping (bottom).

vice. This service is able to wrap any executable code. It exposes a standard interface that takes as input (i) a generic descriptor of the executable command line and (ii) the input parameters and data of this executable. The command line description has to be complete enough to allow dynamic composition of the command line from the list of parameters at the service invocation time and to access the executable and input data files. As a consequence, the executable descriptor contains:

1. The name and access method of the executable. In our current implementation, access methods can be a URL, a Grid File Name (GFN) or a local file name. The wrapper is responsible for fetching the data according to different access methods.

2. The name and access method of the sandboxed files. Sandboxed files are external files such as dynamic libraries or scripts that may be needed for the execution although they do not appear on the command-line.

3. The access method and command-line option of the input data. As our approach is service-based, the actual name of the input data files is not mandatory in the description. Those values will be defined at the execution time. This feature differs from various job description languages used in the task-based middlewares. The command-line option allows the service to dynamically build the actual command-line at the execution time.

4. The command-line option of the input parameters: parameters are values of the command-line that are not files and thus does not have any access method.

5. The access method and command-line option of the output data. This information enables the service to register the output data in a suitable place after the execution.

We gather all this information into an XML file. An example of such as description file is presented in figure 8 . It corresponds to the description of the service crestlines of the workflow of figure 9. It describes the script CrestLines.pl which is available from the server colors. unice.fr and takes 3 input arguments: 2 files (options -im1 and -im2 of the command-line) that are already registered on the grid as GFNs at execution time and 1 parameter (option $-s$ of the command-line). It produces 2 files that will be registered on the grid. It also requires 3 sandboxed files that are available from the server colors.unice.fr.

This generic service highly simplifies application development because it is able to wrap any legacy code with a minimal effort. The application developer only needs writing the executable descriptor for her code to become service aware. But its main advantage is in enabling the sequential services grouping optimization. Indeed, as the workflow enactor has access to the executable descriptors, it is able to dynamically create a virtual service, composing the command lines of the codes to be invoked, and submitting a single job corresponding to this sequence of command lines invocation.

It is important to notice that our solution remains compatible with the services standards. The workflow can still be executed by other enactors, as we did not introduce any new invocation method. Those enactors will make standard service calls (e.g. SOAP ones) to our generic wrapping service.

\section{Results using our enhanced workflow enac- tor prototype}

The goal of this section is to present experimental results that quantify the relevance of the optimizations described above on a real service-based data-intensive application workflow. We first present the design of an enhanced workflow enactor prototype that implements those features and we then evaluate its performance on the EGEE production grid infrastructure.

\subsection{Workflow enactment}

We implemented a prototype of a workflow enactor taking into account the optimizations mentioned in section 3: workflow, data and service parallelism and sequential processors grouping. Our hoMe-made OpTimisEd scUfl enactoR (MOTEUR) prototype was implemented in Java, in order to be platform independent. It is available under CeCILL Public License (a GPL-compatible open source license) at http://www.i3s.unice.fr/ glatard. 


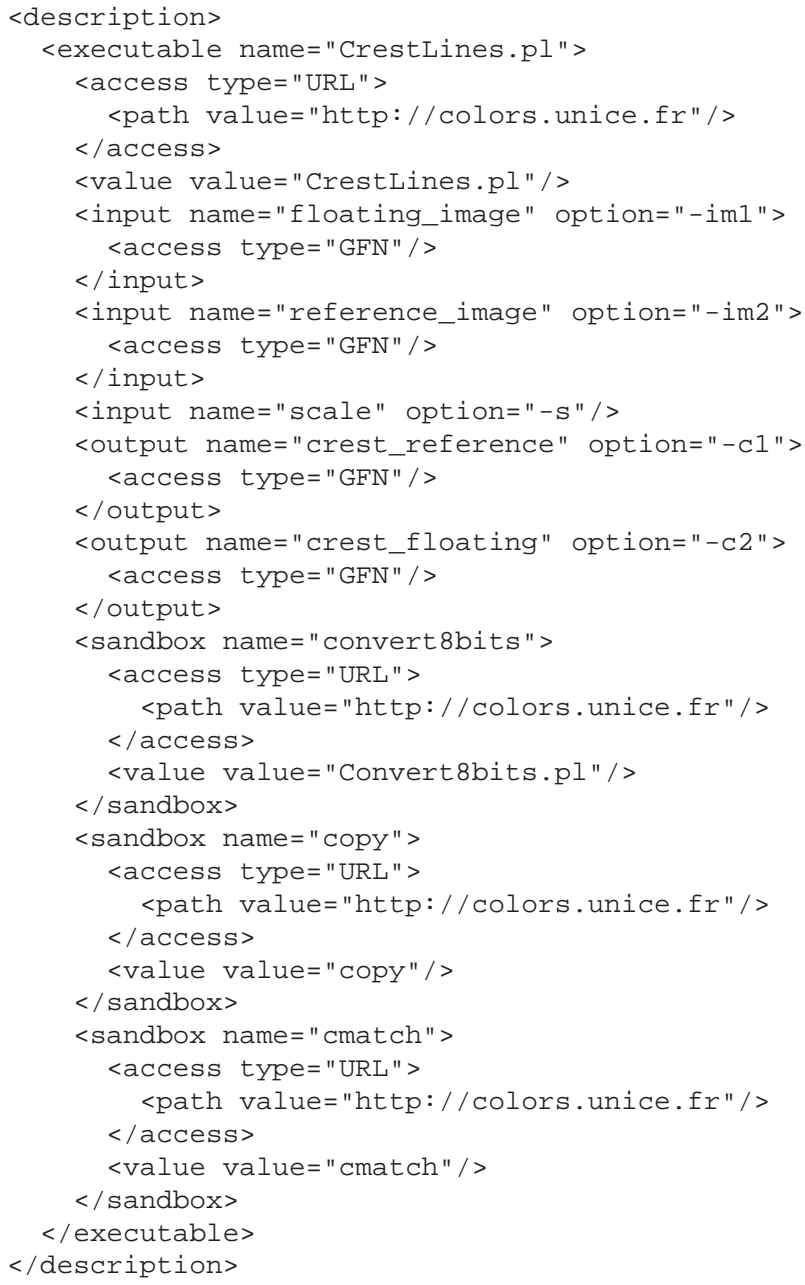

Figure 8. Example of an executable description file.
To our knowledge, this is the only service-based workflow enactor providing all these levels of optimization.

The workflow description language adopted is the Simple Concept Unified Flow Language (Scufl) used by the Taverna workbench [23]. This language is currently becoming a standard in the e-Science community. Apart from describing the data links between the services, the Scufl language allows to define so-called coordination constraints. A coordination constraint is a control link which enforces an order of execution between two services even if there is no data dependency between them. We used those coordination constraints to identify services that require data synchronization.

We developed an XML-based language to be able to describe input data sets. This language aims at providing a file format to save and store the input data set in order to be able to re-execute workflows on the same data set. It simply describes each item of the different inputs of the workflow.

Handling the iteration strategies presented in section 2.2 in a service and data parallel workflow is not straightforward because produced data sets have to be uniquely identified. Indeed they are likely to be computed in a different order in every service, which could lead to wrong dot product computations. Moreover, due to service parallelism, several data sets are processed concurrently and one cannot number all the produced data once computations completed. We have implemented a data provenance strategy to sort out the causality problems that may occur. Attached to each processed data segment is a history tree containing all the intermediate results computed to process it. This tree unambiguously identifies the data.

Finally, MOTEUR is implementing an interface to both Web Services and GridRPC instrumented application code.

\subsection{Bronze Standard application}

We made experiments considering a medical imaging rigid registration application. Medical image registration consists in searching a transformation (that is to say 6 parameters in the rigid case -3 rotation angles and 3 translation parameters) between two images, so that the first one (the floating image) can superimpose on the second one (the reference image) in a common 3D frame. Medical image registration algorithms are a key component of medical imaging workflows.

The application we are working on aims at assessing registration results with a statistical approach called the Bronze-Standard [22]. The goal is basically to compute the registration of a maximum of image pairs with a maximum number of registration algorithms so that we obtain a largely overestimated system to relate the geometry of all the images. As a consequence, the mean registration should be more precise and is called a bronze-standard. It implies that 
we are first able to process registrations of many pairs of images, which is a data-intensive problem.

The workflow of our application is represented on figure 9. The two inputs referencelmage and floating Image correspond to the image sets on which the evaluation is to be processed. The first registration algorithm is crestMatch. Its result is used to initialize the other registration algorithms which are Baladin, Yasmina and PFMatchICP/PFRegister. crestlines is a pre-processing step. Finally, the MultiTransfoTest service is responsible for the evaluation of the accuracy of the registration algorithms, leading to the outputs values of the workflow. This service evaluates the accuracy of a specified registration algorithm by comparing its results with means computed on all the others. Thus, the MultitransfoTest service has to be synchronized: it must be enacted once every of its ancestor is inactive. This is why we figured it with a double square on figure 9.

We chose this particular application because it is a real example of data-intensive workflow in the medical imaging field. Moreover, it embeds a synchronization barrier and thus provides an interesting case of complex service-based workflow.

Input image pairs are taken from a database of injected T1 brain MRIs from the cancer treatment center "Centre Antoine Lacassagne" in Nice, France, courtesy of Dr PierreYves Bondiau. All images are $256 \times 256 \times 60$ and coded on 16 bits, thus leading to a 7.8 MB size per image (approximately $2.3 \mathrm{MB}$ when compressed without loss).

\subsection{Execution platform}

In order to evaluate the relevance of our prototype and to compare real executions to theoretically expected results, we made experiments on the EGEE production grid infrastructure. This platform is a pool of thousands computing (standard PCs) and storage resources accessible through the LCG2 middleware. The resources are assembled in computing centers, each of them running its internal batch scheduler. Jobs are submitted from a user interface to a central Resource Broker which distributes them to the available resources. Not only workflow and service parallelism but also data parallelism make sense on such a grid infrastructure. Indeed, every instance of a service will be submitted to the grid and thus executed on a different processor.

\subsection{Experiments}

We executed our workflow on 3 different inputs data sets, with various sizes, corresponding to the registration of 12 , 66 and 126 image pairs from the above mentioned database, corresponding to images from 1,7 and 25 patients respec-

\begin{tabular}{|c|c|c|c|}
\hline \multirow{2}{*}{ Configuration } & \multicolumn{3}{|c|}{ Computation time (s) } \\
\cline { 2 - 4 } & 12 images & 26 images & 126 images \\
\hline NOP & 32855 & 76354 & 133493 \\
\hline JG & 22990 & 68427 & 125503 \\
\hline SP & 18302 & 63360 & 120407 \\
\hline DP & 17690 & 26437 & 34027 \\
\hline SP+DP & 7825 & 12143 & 17823 \\
\hline SP+DP+JG & 5524 & 9053 & 14547 \\
\hline
\end{tabular}

Table 1. Execution time for each configuration

\begin{tabular}{|c|c|c|}
\hline & $\begin{array}{c}\text { y-intercept } \\
\text { (seconds) }\end{array}$ & $\begin{array}{c}\text { Slope } \\
\text { (s/data sets) }\end{array}$ \\
\hline NOP & 20784 & 884 \\
\hline JG & 11093 & 900 \\
\hline SP & 6382 & 897 \\
\hline DP & 16328 & 143 \\
\hline SP+DP & 6625 & 88 \\
\hline SP+DP+JG & 4310 & 79 \\
\hline
\end{tabular}

Table 2. Y-intercept and slope of the obtained
straight lines, for each configuration

tively on whom acquisitions have been done at several time points to monitor the growth of brain tumors. Each of the input image pair was registered with the 4 algorithms and leads to 6 job submissions, thus producing a total number of 72,396 and 756 job submissions respectively. We submitted each dataset 6 times with 6 different optimization configurations in order to identify the specific gain provided by each optimization.

\subsection{Results}

Figure 10 displays the experimental results we obtained for each of execution configuration mixing Data Parallelism (DP), Service Parallelism (SP) and Job Grouping (JG). The configuration with no optimization (NOP) only includes workflow parallelism. The corresponding numeric values are presented in table 1 with execution times in seconds. The full experiment lead to a total running time of 9 days and 8 hours.

We can notice on figure 10 that the graphical representations of the execution times with respect to the size of the input data set size are almost straight lines. This could be expected as the infrastructure is large enough to support the increasing load. 


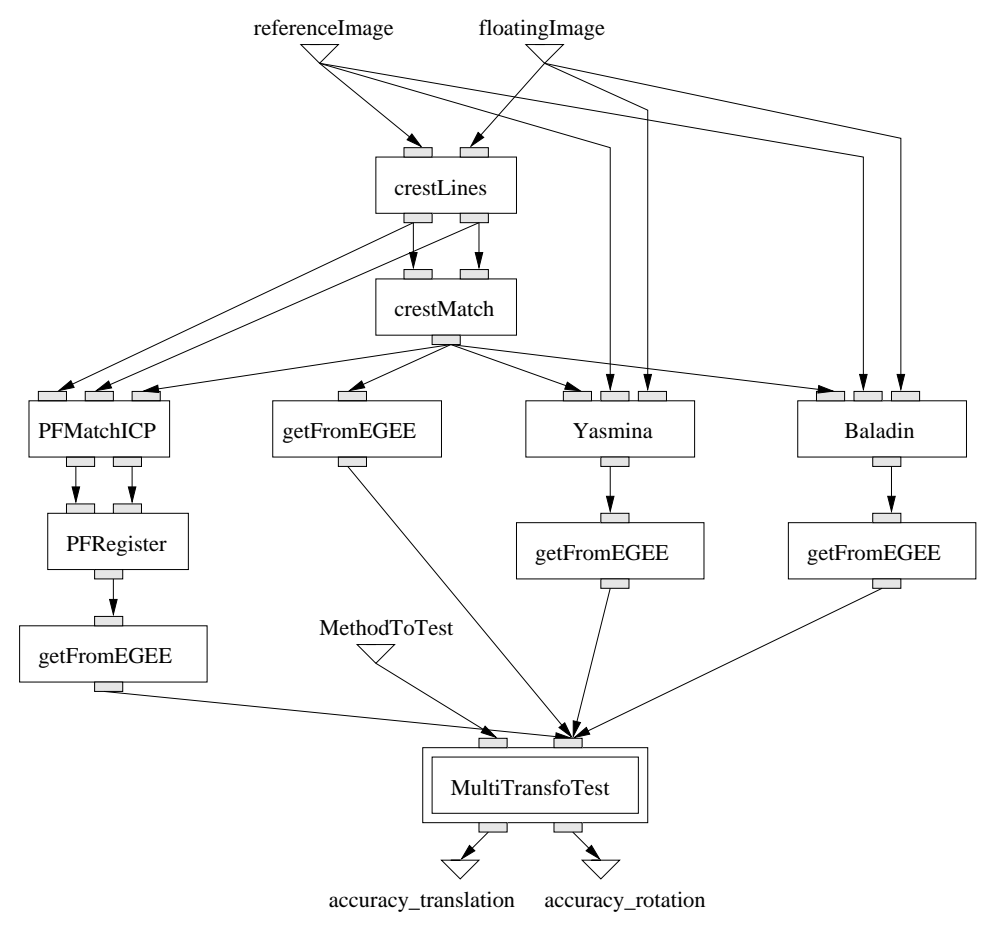

Figure 9. Workflow of the application

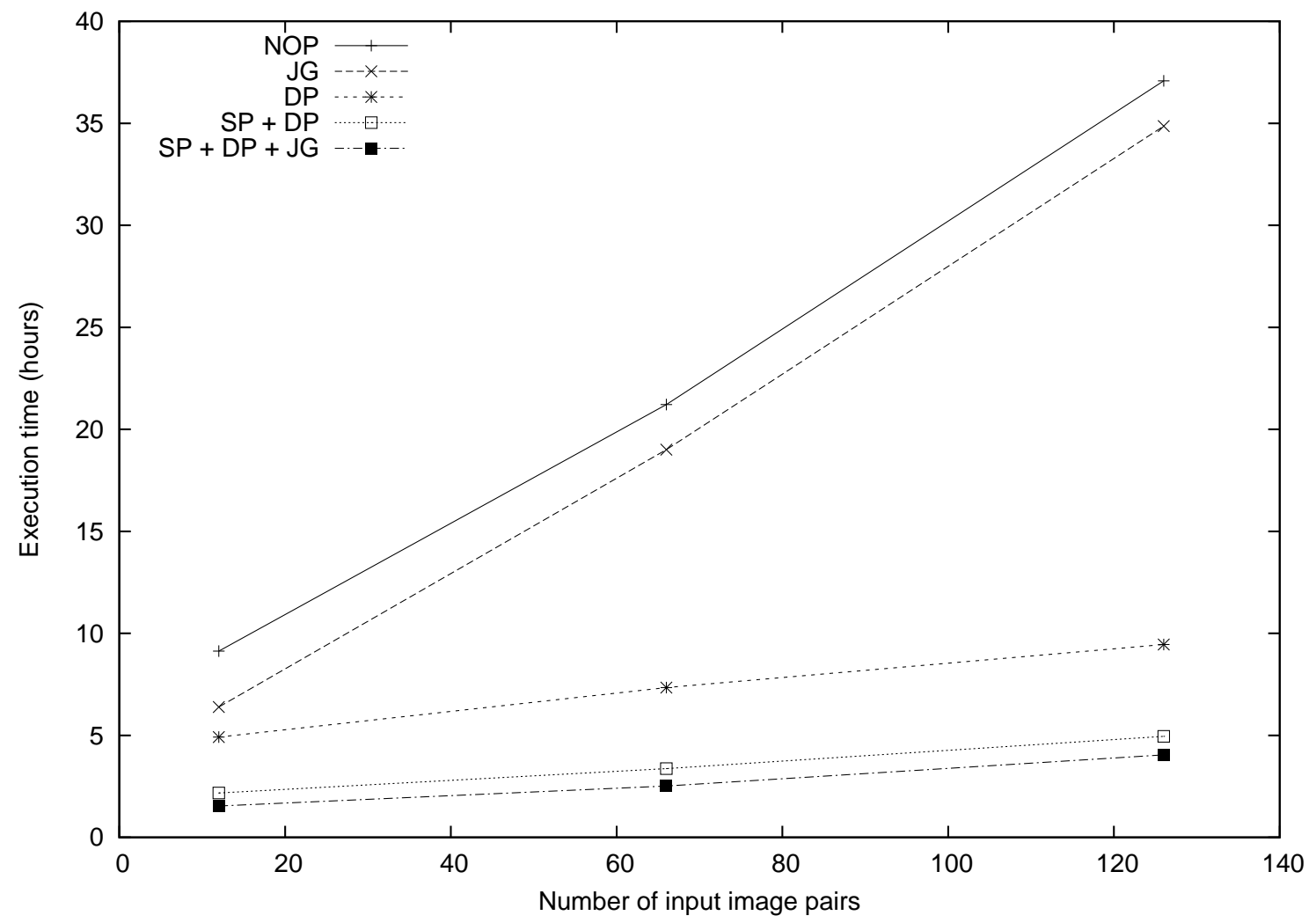

Figure 10. Comparison of the execution times obtained for different optimization configurations 


\section{Discussion}

\subsection{Metrics for the analysis}

To analyze performances, the first relevant metric from the user point of view is the speed-up, measured as the ratio of the execution time over the reference execution time. We also introduce two other metrics, $y$-intercept and slope ratios of the time curves versus input data size, that allow a more precise interpretation of experiments on production grid infrastructures.

Indeed, on multi-users systems, the overhead introduced by submission, scheduling, queuing and data transfers times can be very high (around 10 minutes) and quite variable ( \pm 5 minutes) as we already noticed before. This overhead is measured by the y-intercept value of the curves: this value denotes the time spent for the processing of 0 data set and thus corresponds to the incompressible amount of time required to access the infrastructure. Therefore, we introduce the y-intercept ratio as a metric to compare the influence of methods on the system's overhead. This ratio compares the y-intercept value of the analyzed line with the one of a reference line.

Besides, given the linear aspect of the execution time curves, we approximate them as lines by linear regression. The slope of these regression lines measure the data scalability of the grid, that is to say its ability to process huge data sets with the same level of performance. Consequently, we introduce the slope ratio as a metric to compare the influence of methods on the infrastructure data scalability. This ratio compares the slope value of the analyzed line with the one of a reference line. The higher the number of data to process, the more important the slope ratio parameter is compared to the y-intercept one.

In case of low latency infrastructures such as local clusters, the y-intercept ratio would be meaningless because it would be close to 0 . This metric is thus more specific to production grid infrastructures such as the one we described in section 4.3.

In the following sections, we use the slope and yintercept ratios in addition to the speed-up metric. For each configuration, we reported the y-intercept and the slope of the lines in table 2 . Those values were obtained by linear regressions on measurements displayed in table 1 . We relate our experimental results to the theoretical ones that we presented in section 3.5. As our data set is quite homogeneous (all the images have the same size), we make the hypothesis of constant execution times and thus refer to the results presented in the last paragraph of section 3.5.4 and in particular to $S_{D P}, S_{S P}$ and $S_{S D P}$. For our application, $n_{W}$ is 5 and $n_{D}$ is either 12,66 or 126 , depending on the input data set.

\subsection{Impact of the data and service paral- lelism}

DP versus NOP. Given the data-intensive nature of the application, the first level of parallelism to enable to improve performances is data parallelism. In this case, the last paragraph of section 3.5.4 predicted a speed-up $S_{D}=n_{D}$. We obtain speed-ups of $1.86,2.89$ and 3.92 for $n_{D}=12$, 66 and 126 image pairs respectively. This speed-up is effectively growing with the number of input images as predicted by the theory, although it is lower than expected. Indeed, this experiment shows that the system variability (on transfer and queuing time in particular) and the increasing load of the middleware services on a production infrastructure cannot be neglected.

To go further in the analysis, we can compute from table 2 that in this case, data parallelism leads to a slope ratio of 6.18 and to a y-intercept ratio of 1.27. Data parallelism thus mainly influences the slope ratio. It is coherent as this metric is designed to evaluate the data scalability of the system. Although a higher slope ratio could be expected on a dedicated system to some extent (until the number of dedicated resources is reached), we can see that in our experiment the grid infrastructure smoothly accepts the increasing load (no saturation effect). This is interesting for applications such as the Bronze Standard that needs the highest number of data to be processed as possible.

$(\mathrm{DP}+\mathrm{SP})$ versus DP. One can notice is that service parallelism does introduce a significant speed-up even if data parallelism is enabled. Indeed, it leads to a speed-up of 2.26 , 2.17 and 1.90 for 12,66 and 126 image pairs respectively whereas the theory predicted a speed-up of $S_{S D P}=1$. This result can be justified by noticing that the constant times hypothesis may not hold on such a production infrastructure, as already suggested in section 3.5.4. On a traditional cluster infrastructure, service parallelism would be of minor importance whereas it is a very important optimization on the production infrastructure we used.

Moreover, we can then notice that in case of data parallelism, service parallelism leads to a slope ratio of 1.62 and to a y-intercept ratio of 2.46. This is another argument which demonstrates that service parallelism is particularly important on production infrastructures. On traditional clusters indeed, y-intercept values may be close to 0 and such systems would therefore be less impacted by a reduction of this metric.

\subsection{Impact of the job grouping}

JG vs NOP. The speed-up introduced by job grouping is $1.43,1.12$ and 1.06 for 12,66 and 126 image pairs respectively. It leads to a slope ratio of 0.98 and to a y-intercept 
ratio of 1.87 . Job grouping only influences the y-intercept ratio. It is coherent because it has been designed to lower the system's overhead which is evaluated by the y-intercept value.

$(\mathbf{J G}+\mathbf{S P}+\mathbf{D P})$ vs $(\mathbf{S P}+\mathbf{D P})$ In addition to data and service parallelism, job grouping introduces a speed-up of $1.42,1.34$ and 1.23 for 12,66 and 126 image pairs respectively. It leads to a slope ratio of 1.11 and to a y-intercept ratio of 1.54 . Here again, job grouping mainly improves the y-intercept ratio, which is coherent with the expected behavior.

We can thus conclude that job grouping effectively addresses the problem for which it as been designed as it leads to a significant reduction of the system's overhead.

\subsection{Optimization perspectives}

The y-intercept and slope values are able to quantify how an application could be improved, without any reference to the scale of the infrastructure. Indeed, an ideal system would have a null slope and a close to zero y-intersect.

The $y$-intercept value of $\mathrm{DP}+\mathrm{SP}+\mathrm{JG}$ quantifies the potential overhead reduction that could be targeted. In the future, we plan to address this problem by grouping jobs of a single service, thus finding an trade-off between data parallelism and the system's overhead.

Besides, the slope value of $\mathrm{DP}+\mathrm{SP}+\mathrm{JG}$ quantifies the potential data scaling improvement that could be targeted. On an ever-loaded production infrastructure, middleware services such as the user interface or the resource broker may be critical bottlenecks. The theoretical modeling does not take into account these limitations. A probabilistic modeling considering the variable nature of the grid infrastructure is probably an interesting future path to explore for further optimizing this value [12].

\subsection{Conclusions}

Grids have a very interesting potential for processing data intensive applications and composing new applications from services wrapping application code. However, the level of flexibility introduced by the service based approach also lowers the computations efficiency.

In this paper we have reviewed several optimization strategies that can be exploited to efficiently process such applications. We proposed a method for grouping job submissions in a service-based workflow. We designed a complete prototype of an optimized workflow enactor taking into account workflow, services and data parallelism and which is able to group sequential jobs in order to speedup the execution on a grid infrastructure. The implemented prototype is able to deal with existing standard workflows and services description languages. To our knowledge no other workflow enactor does exploit all these strategies today. The modeling of the overall execution times demonstrates that in different scenarios, the workflow manager never leads to performance drops and that it is particularly efficient for dealing with data-intensive applications. This is confirmed by an experiment on a medical image registration application which precisely quantifies the impact of these optimizations. In addition to the traditional speed-up metric, we introduced the y-intercept and slope ratios which are particularly suited for the interpretation of optimizations on production grids.

We have plans for further optimizing the workflow engine. In particular, we are thinking of an optimal strategy to adapt the jobs' granularity to the grid load and a probabilistic modeling of the ever-changing production grid infrastructure.

\section{Acknowledgment}

This work is partially funded by the French research program "ACI-Masse de données" (http://acimd.labri.fr/), AGIR project (http://www.aci-agir.org/). We are grateful to the EGEE European project for providing the grid infrastructure and user assistance.

\section{References}

[1] D. Arnold, S. Agrawal, S. Blackford, J. Dongarra, M. Miller, K. Seymour, K. Sagi, Z. Shi, and S. Vadhiyar. Users' Guide to NetSolve V1.4.1. Technical Report ICL-UT-02-05, University of Tennessee, Knoxville, jun 2002.

[2] J. Blythe, S. Jain, E. Deelman, Y. Gil, K. Vahi, A. Mandal, and K. Kennedy. Task Scheduling Strategies for Workflowbased Applications in Grids. In CCGrid, Cardiff, UK, 2005.

[3] F. Cappello, F. Desprez, M. Dayde, E. Jeannot, Y. Jegou, S. Lanteri, N. Melab, R. Namyst, P. Vicat-Blanc Primet, O. Richard, E. Caron, J. Leduc, and G. Mornet. Grid'5000: A Large Scale, Reconfigurable, Controlable and Monitorable Grid Platform. In 6th IEEE/ACM International Workshop on Grid Computing (Grid'2005), Seattle, Washington, USA, nov 2005.

[4] E. Caron, B. Del-Fabbro, F. Desprez, E. Jeannot, and J.M. Nicod. Managing Data Persistence in Network Enabled Servers. Scientific Programming Journal, 2005.

[5] E. Caron, F. Desprez, F. Lombard, J.-M. Nicod, M. Quinson, and F. Suter. A Scalable Approach to Network Enabled Servers. In 8th International EuroPar Conference (), volume 2400 of LNCS, pages 907-910, Paderborn, Germany, aug 2002. Springer-Verlag.

[6] H. Casanova, A. Legrand, D. Zagorodnov, and F. Berman. Heuristics for Scheduling Parameter Sweep Applications in Grid Environments. In 9th Heterogeneous Computing Workshop $(H C W)$, pages 349-363, Cancun, may 2000. 
[7] D. Churches, B. S. Sathyaprakash, M. Shields, I. Taylor, and I. Wand. A Parallel Implementation of the Inspiral Search Algorithm using Triana. In Proceedings of the UK e-Science All Hands Meeting (), Nottingham, UK, sep 2003.

[8] European IST project of the FP6, Enabling Grids for E-sciencE, apr. 2004-mar. 2006. http: //www. eu-egee.org/.

[9] I. Foster. Globus Toolkit Version 4: Software for ServiceOriented Systems. In International Conference on Network and Parallel Computing (IFIP), volume 3779, pages 2-13. Springer-Verlag LNCS, 2005.

[10] I. Foster, C. Kesselman, J. Nick, and S. Tuecke. The Physiology of the Grid: An Open Grid Services Architecture for Distributed Systems Integration. Technical report, Open Grid Service Infrastructure WG, Global Grid Forum, June 2002.

[11] T. Glatard, J. Montagnat, and X. Pennec. Grid-enabled workflows for data intensive medical applications. In 18th IEEE International Symposium on Computer-Based Medical Systems (ISCBMS), jun 2005.

[12] T. Glatard, J. Montagnat, and X. Pennec. Probabilistic and dynamic optimization of job partitioning on a grid infrastructure. In 14th euromicro conference on Parallel, Distributed and network-based Processing, pages 735-745, Montbliard-Sochaux, France, Feb. 2006.

[13] gLite middleware. http: //www.gLite.org.

[14] R. Irani and S. J. Bashna. AXIS: Next Generation Java SOAP. Wrox Press, may 2002.

[15] P. Kacsuk, A. Goyeneche, T. Delaitre, T. Kiss, Z. Farkas, and T. Boczko. High-Level Grid Application Environment to Use Legacy Codes as OGSA Grid Services. In Proceedings of the Fifth IEEE/ACM International Workshop on Grid Computing (GRID '04), pages 428-435, Washington, DC, USA, 2004. IEEE Computer Society.

[16] LCG2 middleware. http://lcg.web.cern.ch/LCG/activities/middleware.html.

[17] P. Lord, P. Alper, C. Wroe, and C. Goble. Feta: A lightweight architecture for user oriented semantic service discovery. In European Semantic Web Conference (), 2005.

[18] B. Ludscher, I. Altintas, C. Berkley, D. Higgins, E. Jaeger, M. Jones, E. A. Lee, J. Tao, and Y. Zhao. Scientific Workflow Management and the Kepler System. Concurrency and Computation: Practice \& Experience, 2005.

[19] J. Montagnat, F. Bellet, H. Benoit-Cattin, V. Breton, L. Brunie, H. Duque, Y. Legré, I. Magnin, L. Maigne, S. Miguet, J.-M. Pierson, L. Seitz, and T. Tweed. Medical images simulation, storage, and processing on the european datagrid testbed. Journal of Grid Computing, 2(4):387-400, Dec. 2004.

[20] H. Nakada, S. Matsuoka, K. Seymour, J. Dongarra, C. Lee, and H. Casanova. A GridRPC Model and API for End-User Applications. Technical report, Global Grid Forum (GGF), jul 2005.

[21] National Research Grid Initiative (NAREGI). http: //www.naregi.org.

[22] S. Nicolau, X. Pennec, L. Soler, and N. Ayache. Evaluation of a New 3D/2D Registration Criterion for Liver RadioFrequencies Guided by Augmented Reality. In International Symposium on Surgery Simulation and Soft Tissue Modeling
(IS4TM'03), volume 2673 of LNCS, pages 270-283, Juanles-Pins, 2003. INRIA Sophia Antipolis, Springer-Verlag.

[23] T. Oinn, M. Addis, J. Ferris, D. Marvin, M. Senger, M. Greenwood, T. Carver, K. Glover, M. R. Pocock, A. Wipat, and P. Li. Taverna: A tool for the composition and enactment of bioinformatics workflows. Bioinformatics journal, 17(20):3045-3054, 2004.

[24] Open Science Grid (OSG). http: / / www. opensciencegrid.org.

[25] M. Senger, P. Rice, and T. Oinn. Soaplab - a unified Sesame door to analysis tool. In UK e-Science All Hands Meeting (), pages 509-513, Nottingham, sep 2003.

[26] Y. Tanaka, H. Nakada, S. Sekiguchi, T. Suzumura, and S. Matsuoka. Ninf-G: A Reference Implementation of RPCbased Programming Middleware for Grid Computing. Journal of Grid Computing (JGC), 1(1):41-51, 2003.

[27] I. Taylor, M. Shields, I. Wand, and R. Philp. Grid Enabling Applications Using Triana. In Workshop on Grid Applications and Programming Tools (). Held in Conjunction with GGF8, 2003.

[28] I. Taylor, I. Wand, M. Shields, and S. Majithia. Distributed computing with Triana on the Grid. Concurrency and Computation: Practice \& Experience, 17(1-18), 2005.

[29] R. A. Van Engelen and K. A. Gallivan. The gSOAP Toolkit for Web Services and Peer-to-Peer Computing Networks. In Proceedings of the 2nd IEEE/ACM International Symposium on Cluster Computing and the Grid (CCGRID '02), page 128, Washington, DC, USA, 2002. IEEE Computer Society.

[30] W. World Wide Web Consortium. Web Services Description Language (WSDL) 1.1, mar 2001.

[31] J. Yu and R. Buyya. A taxonomy of scientific workflow systems for grid computing. ACM SIGMOD Record, 34(3):4449, Sept. 2005.

[32] J. Zhao, C. Goble, R. Stevens, D. Quan, and M. Greenwood. Using Semantic Web Technologies for Representing e-Science Provenance. In Third International Semantic Web Conference (ISWC2004), pages 92-106, Hiroshima, nov 2004. 